\title{
A Rare Case Mimicking Collecting System Tumor: Antopol-Goldman Lesion
}

\author{
(1) Kamil Gökhan Şeker MD, (1) Emre Şam MD, (1) Osman Özdemir MD, (1) İsmail Yiğitbaşı MD, (1) İsmail Evren MD, \\ (D) Ekrem Güner MD, (D) Volkan Tuğcu MD \\ University of Health Sciences, Bakırköy Dr. Sadi Konuk Training and Research Hospital, Clinic of Urology, Istanbul, Turkey
}

\begin{abstract}
Antopol-Goldman (AG) lesion is a benign condition characterized by flank pain, hematuria, and radiologically detected filling defect in the renal pelvis. It is clinically important because it is confused with renal parenchymal and collecting system tumors. In this case report, we present a 75-year-old male patient who was evaluated with the suspicion of renal pelvis tumor but was diagnosed with AG lesion and treated conservatively after excluding malignancy with radiology, endoscopy, and pathology. Our aim is to emphasize the importance of diagnostic flexible ureterorenoscopy before planning radical treatment in patients with hematuria, flank pain, and radiological signs of filling defect in the renal pelvis.
\end{abstract}

Keywords: Subepithelial pelvic hematoma, Antopol-Goldman lesion, collecting system tumor, hematuria

\section{Introduction}

Antopol-Goldman (AG) lesion, also called subepithelial pelvic hematoma, was first described by Antopol and Goldman in 1948. They published a report of 7 patients who underwent radical nephrectomy for a diagnosis of collecting system and/ or renal tumor (1).

AG lesions are benign and diagnosed based on hematuria, flank pain, and radiologically detected filling defect in the renal pelvis. It regresses clinically and radiologically with a conservative therapeutic approach. The objective of this case report is to present a 75-year-old male patient who was evaluated with the suspicion of a right pelvis tumor but was diagnosed with AG lesion and managed with conservative treatment after excluding malignancy with radiology, endoscopy, and pathology.

\section{Case Report}

A 75-year-old male patient presented to the emergency department with complaints of painless gross hematuria with blood clots. His medical history was unremarkable except for a myeloproliferative disorder. He had no history of trauma, tobacco consumption, or use of anticoagulant or antiaggregant agents. His hemoglobin level was $8.09 \mathrm{~g} / \mathrm{dL}$, platelet count was 56,400, and renal function tests were high (creatinine: $3.22 \mathrm{mg} / \mathrm{dL}$, urea: $79 \mathrm{mg} / \mathrm{dL}$ ). In previous tests, his creatinine level was $2.62 \mathrm{mg} / \mathrm{dL}$ and it was considered chronic kidney disease. On ultrasound (US) examination, the right renal pelvis and calyces had a distinct appearance and a lesion
$40 \times 40 \mathrm{~mm}$ in size invading the lower calyx in particular was observed. Unenhanced computed tomography (CT) of the abdomen performed due to elevated creatinine level revealed a $40 \times 45 \mathrm{~mm}$ space-occupying heterogeneous lesion in the right renal pelvis (Figure 1). The average Hounsfield unit value was 61.68. A collecting system tumor or hematoma related to the hemorrhage was not clearly distinguished. Although a right nephroureterectomy was initially planned, we decided to confirm the diagnosis with endoscopy because of the disrupted kidney function. Diagnostic cystoscopy and right diagnostic ureterorenoscopy were carried out. There was no tumoral structure in the bladder, but hematuric jet flow was observed from the right ureteral orifice. No space-occupying mass in the ureteral segments or renal pelvis was seen in ureterorenoscopy. There were hyperemic areas in the renal pelvis (Figure 2). A punch biopsy was taken from these areas. Histopathological results showed no malignancy, thus transitional cell carcinoma of the collecting system was excluded (Figure 3).

With a clinical diagnosis of AG lesion, we opted for a conservative treatment approach with analgesic agents and bed rest. During the follow-up period, his hematuria improved and abdominal CT at 3 months showed that the space-occupying lesion in the right renal pelvis had disappeared (Figure 4). Written informed consent was obtained from the patient.

\section{Discussion}

AG lesion is a rare benign disorder that is difficult to diagnose because it is easily mistaken for renal parenchymal and

Address for Correspondence: Kamil Gökhan Şeker MD, University of Health Sciences, Bakırköy Dr. Sadi Konuk Training and Research Hospital, Clinic of Urology, İstanbul, Turkey E-mail: gkhnseker@hotmail.com ORCID-ID: orcid.org/0000-0003-4449-9037 
collecting system tumors. Although it occurs in every age group regardless of gender, it is more common in the elderly. However, 1 neonatal case and a few cases in patients younger than 40 years old have also been reported $(2,3)$.

Although several factors are believed to be involved in the etiology, it has not been fully elucidated. Factors such as the usage of analgesics and anticoagulants have been implicated (4). Besides these factors, trauma, underlying congenital malformations, diabetes, hypertension, drug addiction, and amyloidosis have been also reported as associated factors (5). Although none of these factors were present in our case, we suspect that the lesion may have been caused by thrombocytopenia associated with the patient's known myeloproliferative disorder.

Medical history and especially radiological examination are supportive of the diagnosis in patients with AG lesions,, who present with hematuria. The most common symptoms of AG lesion are hematuria and flank pain $(2,5)$. Unfortunately, diagnosing AG lesions with radiological methods alone is difficult. Therefore, patients are usually diagnosed based on histopathological examination of specimens obtained during partial or radical nephrectomy. Although a heterogeneous space-occupying lesion may be detected in US, CT should be carried out in order to define the hemorrhagic component with its heterogeneous hyperdense appearance (4). In our case, a space-occupying lesion extending from the renal pelvis to the lower calyx was diagnosed with US and verified with unenhanced abdominal CT, which was preferred due to the presence of the renal failure.

Following the description of AG lesion in 1948, the increase in reported cases led urologists to implement medical treatments and follow-up instead of urgent surgery. Cardin et al. (4) first reported the successive treatment of patients with AG lesion using a conservative approach. They recommended a conservative follow-up approach in patients who had a history of coagulopathy and anticoagulant agent use, a negative ureterorenoscopic examination, and improvement in hematuria symptoms. In recent years, the diagnosis of renal pelvic lesions has been facilitated by increasing use of flexible ureterorenoscopy, and thus conservative approaches have gained momentum. Altay et al. (2) did not detect any tumoral structure in the urinary system with flexible ureterorenoscopy

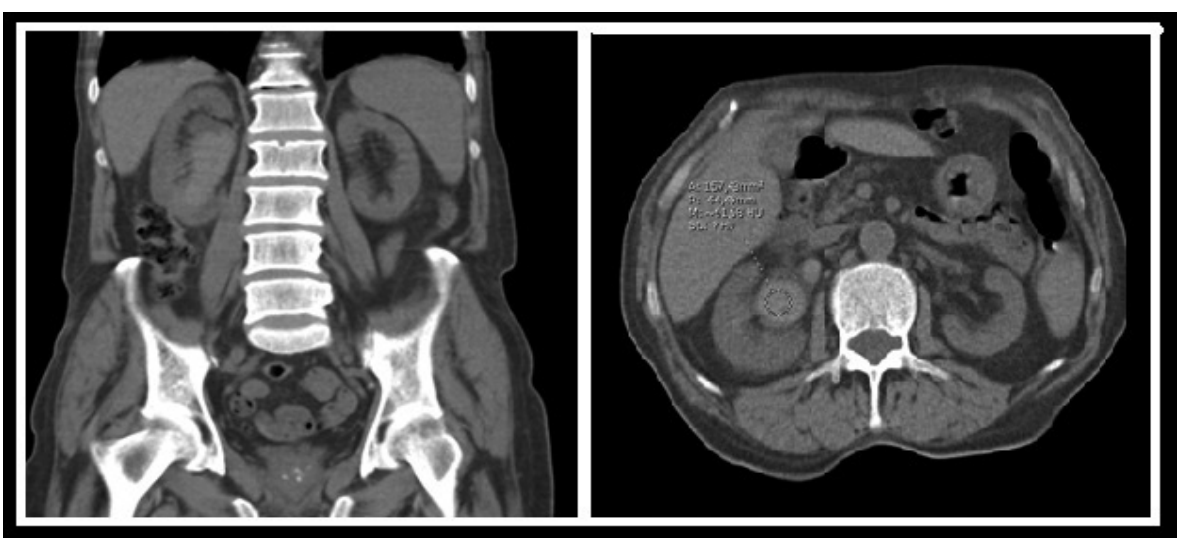

Figure 1. Hyperdense space-occupying lesion extending from the right renal pelvis to the lower calyx in the coronal and axial sections of the computed tomography

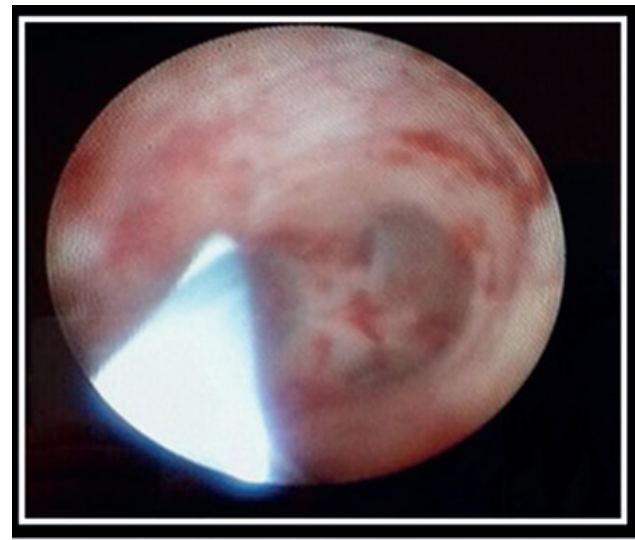

Figure 2. Hyperemia and hemorrhagic loci in the renal pelvis in right diagnostic ureterorenoscopy

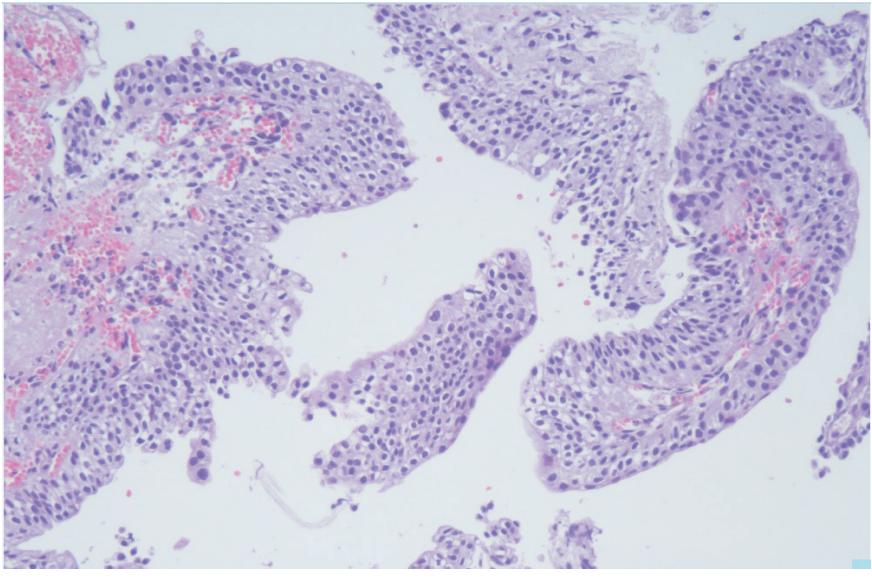

Figure 3. Papillary urothelial hyperplasia that looks like normal epithelium with hematoxylin and eosin stain in $200 x$ magnification

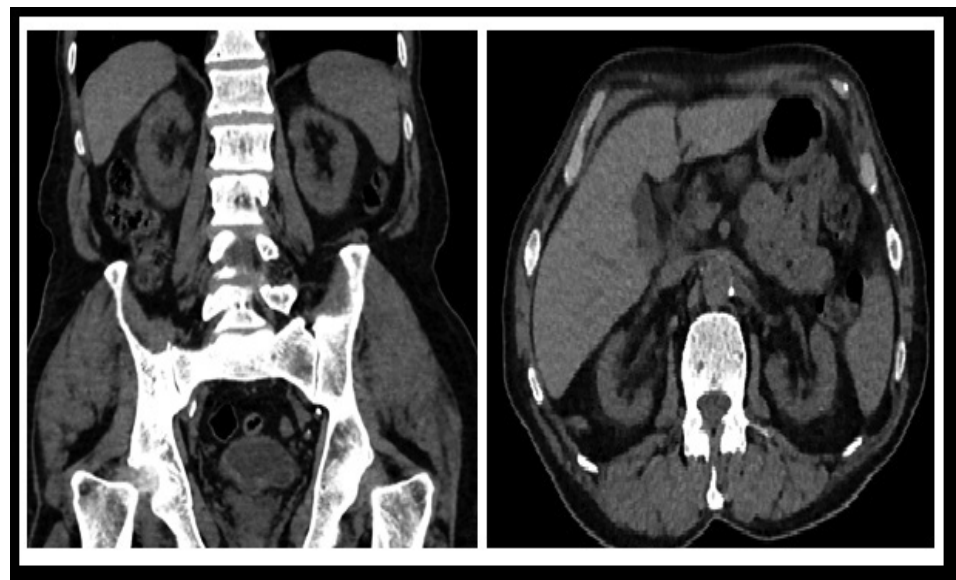

Figure 4. Regression of the mass in follow-up computed tomography 
in an 80-year-old patient who presented with gross hematuria. They diagnosed the patient with AG lesion and treated the patient with tranexamic acid and fibrinolytics for 4 weeks after insertion of a double J catheter. They reported that the lesion had disappeared in the follow-up imaging done at 3 months. Gayer et al. (6) diagnosed AG lesions in 7 patients with coagulopathy, and reported that the lesions improved with conservative follow-up. They emphasized the importance of informing the radiologist during the request for radiological imaging. In our case, after the radiologist's initial report in favor of hematoma, the flexible ureterorenoscopy was carried out and no space-occupying lesion was detected in the renal pelvis. The lesion regressed during follow-up.

In conclusion, AG lesion should be kept in mind in patients with radiological findings of filling defect in the renal pelvis, and if possible, diagnostic flexible ureterorenoscopy should be performed to avoid radical surgery and its complications.

\section{Ethics}

Informed Consent: Written informed consent was obtained from the patient.

Peer-review: Externally and internally peer-reviewed.

\section{Authorship Contributions}

Surgical and Medical Practices: K.G.Ş., E.Ş., O.Ö., I.Y., I.E., E.G., V.T., Concept: K.G.Ş., E.Ş., O.Ö., İ.Y., Design: İ.Y., İ.E., E.G., V.T.,
Data Collection or Processing: E.Ş., O.Ö., I.Y., İ.E., Analysis or Interpretation: K.G.Ş., E.Ş., O.Ö., V.T., Literature Search: K.G.Ş., I.E., E.G., V.T., Writing: K.G.Ş., E.Ş., O.Ö., I.Y., İ.E., E.G., V.T.

Conflict of Interest: No conflict of interest was declared by the authors.

Financial Disclosure: The authors declared that this study received no financial support.

\section{References}

1. Antopol W, Goldman L. Subepithelial hemorrhage of renal pelvis simulating neoplasm. Urol Cutaneous Rev 1948;52:189-195.

2. Altay B, Barışık CC, Erkurt B, Kiremit MC. Subepithelial pelvic hematoma of the kidney (Antopol-Goldman Lesion). Turk J Urol 2015;41:48-50.

3. Chan IH, Lam WW, Wong KK, Tam PK. Renal pelvis haematoma causing pelviureteric obstruction: a first case of Antopol-Goldman lesion in a neonate. J Paediatr Child Health 2010;46:361-362.

4. Cardin AL, Marshall J, Bhatt S, et al. Antopol-Goldman lesion of the kidney diagnosed byradiology: a casereport of observation. Acta Radiol 2008;49:715-717.

5. Eccher A, Brunelli M, Gobbo S, et al. Subepithelial pelvic hematoma (Antopol-Goldman lesion) simulating renal neoplasm: report of a case and review of the literature. Int J SurgPathol 2009;17:264-267.

6. Gayer G, Desser TS, Hertz M, et al. Spontaneous suburothelial hemorrhage in coagulopathic patients: CT diagnosis. AJR Am J Roentgenol 2011;197:887-890. 